\title{
A phase II clinical trial of palonosetron for the management of delayed vomiting in gynecological cancer patients receiving paclitaxel/carboplatin therapy
}

\author{
ERIKO TAKATORI, TADAHIRO SHOJI, YUKI MIURA, MIYUKI NAGAO, ANNA TAKADA, \\ TAKAYUKI NAGASAWA, HIDEO OMI, MASAHIRO KAGABU, TATSUYA HONDA and TORU SUGIYAMA \\ Department of Obstetrics and Gynecology, Iwate Medical University School of Medicine, Morioka, Iwate 020-8505, Japan
}

Received September 10, 2014; Accepted November 1, 2014

DOI: $10.3892 / \mathrm{mco} .2015 .484$

\begin{abstract}
There are currently no studies demonstrating the effects of palonosetron on delayed chemotherapy-induced nausea and vomiting (CINV) in gynecological cancer patients receiving chemotherapy with moderately emetogenic chemotherapeutic agents. We conducted a phase II clinical trial to assess the efficacy and safety of palonosetron in patients receiving paclitaxel/carboplatin (TC) therapy. The study population consisted of 42 patients who had been diagnosed with gynecological malignancies and treated with TC. On day 1 , $0.75 \mathrm{mg} /$ body palonosetron and $19.8 \mathrm{mg} /$ body dexamethasone were administered intravenously immediately prior to TC therapy. Dexamethasone in daily doses of $6.6 \mathrm{mg} /$ body was also administered intravenously on days 2 and 3 . The efficacy and safety of palonosetron + dexamethasone were evaluated by the self-completion method using the Multinational Association of Supportive Care in Cancer Antiemesis Tool during an observation period lasting from day 1 through day 8 of the initial cycle of TC therapy. The severity of the nausea was assessed using a visual analog scale. During the acute (0-24 h), delayed (24-96 h) and overall (0-96 h) periods, the complete response rates were $95.2,90.5$ and $85.7 \%$, respectively, whereas the complete control rates were 90.5, 85.7 and $78.6 \%$, respectively. Grade $\geq 2$ constipation and diarrhea developed in 1 patient $(2.4 \%)$ each. The palonosetron + dexamethasone regimen proved to be effective for delayed CINV in gynecological cancer patients receiving TC therapy. This combined antiemetic regimen was associated with only mild adverse reactions and may serve as supportive therapy, allowing cancer chemotherapy to be continued while maintaining an adequate quality of life.
\end{abstract}

Correspondence to: Dr Tadahiro Shoji, Department of Obstetrics and Gynecology, Iwate Medical University School of Medicine, 19-1 Uchimaru, Morioka, Iwate 020-8505, Japan

E-mail: tshoji@iwate-med.ac.jp

Key words: palonosetron, chemotherapy-induced nausea and vomiting, paclitaxel/carboplatin therapy, gynecological cancer, phase II study

\section{Introduction}

It is generally recognized that cancer chemotherapy-induced nausea and vomiting (CINV) occurs more commonly in women compared to men and also tends to occur more often in younger compared to older patients (1). CINV compromises the quality of life (QOL) of the patients and reduces treatment compliance, leading even to treatment refusal or discontinuation (2). Therefore, it is crucial to establish an antiemetic therapy capable of fully preventing, or at least controlling, CINV.

A two-drug antiemetic regimen consisting of a first-generation 5-hydroxytryptamine 3 receptor antagonist (5-HT3RA), such as granisetron, together with dexamethasone, was mainly prescribed in the 1990s. This combined drug regimen was shown to be highly effective in the management of acute CINV (0-24 h following anticancer drug administration), but was not sufficiently effective for the relief of delayed CINV (24-120 h following anticancer drug administration) (3). Later, in the early 21 th century, the neurokinin 1 receptor antagonist (NK1RA) aprepitant and the second-generation 5-HT3RA palonosetron became available for clinical use. These antiemetics are also reportedly effective for the relief of delayed CINV $(4,5)$. The regimen paclitaxel/carboplatin (TC) is currently extensively administered as chemotherapy for gynecological cancers; however, there are currently no reports available on the effects of palonosetron on delayed CINV. Therefore, we conducted a phase II clinical trial to corroborate the efficacy and safety of palonosetron + dexamethasone in patients receiving TC therapy.

\section{Subjects and methods}

Patients. The study population comprised 42 patients who had been diagnosed with gynecological malignancies and treated with TC at Iwate Medical University between January, 2011 and March, 2013. For the assessment of therapeutic response, the following definitions were employed to describe CINV outcomes: i) complete response (CR), complete arrest/prevention of vomiting (no vomiting-related events, no antiemetic treatment -irrespective of the severity of nausea- and no rescue therapy); and ii) complete control 
(CC), complete control of vomiting-related episodes (no antiemetic treatment, only mild nausea, and no rescue therapy). The efficacy and safety of palonosetron + dexamethasone medications were evaluated by the self-completion method using the Multinational Association of Supportive Care in Cancer (MASCC) Antiemesis Tool (6) during an observation period lasting from day 1 through day 8 of the initial cycle of TC therapy. The severity of nausea was assessed using a visual analog scale (VAS) and scored as follows: mild, 1-2; moderate, 3-4; and severe, $\geq 5$. The primary endpoint was the $\mathrm{CR}$ rate for the delayed period (24-96 $\mathrm{h}$ after initiation of TC therapy) and the secondary endpoints were as follows: i) CR rates for the acute period (0-24 $\mathrm{h}$ after the initiation of TC therapy) and for the overall period (0-96 h); ii) CC rates for the acute, delayed and overall periods after the initiation of TC therapy; iii) the severity of nausea during the acute, delayed and overall periods after the initiation of TC therapy; and iv) adverse reactions occurring during the observation period.

Subgroup analyses were performed to comparatively assess the delayed period CR rate in the following strata: age $\left(\geq 55\right.$ vs. $<55$ years), body surface area $\left(\geq 1.47 \mathrm{vs} .<1.47 \mathrm{~m}^{2}\right)$, performance status (PS) (0 vs. 1-2) and complications (present vs. absent).

Inclusion criteria. Patients meeting all the following criteria were enrolled in this study: i) Aged $\geq 20$ years at the time of enrollment; ii) diagnosed with gynecological cancer; iii) naïve to cancer chemotherapy or previous treatment with a single antineoplastic agent less potent than 'drugs with slight emetogenic risk' according to the NCCN Clinical Prectice Guidelines in Oncology (Antiemesis Version 4, 2009) (7) (however, use of hormone preparations for endocrinotherapy and use of antineoplastic agents for purposes other than cancer treatment were not considered as chemotherapy); iv) scheduled to receive the tri-weekly TC therapy regimen (paclitaxel $175 \mathrm{mg} / \mathrm{m}^{2}$ and carboplatin area under the curve 6 administered on day 1); v) patients whose bone marrow and hepatic and renal functions were maintained and whose laboratory test values within 8 days prior to enrollment, including the day of enrollment, fulfilled the criteria of white blood cell count $\geq 3,000 / \mathrm{mm}^{3}$, aspartate aminotransferase and alanine aminotransferase $<100$ IU/1 [or grade $\leq 3$ according to the Common Terminology Criteria for Adverse Events (CTCAE) v4.0 (8), in the case of hepatic metastasis evident on imaging] and creatinine clearance $\geq 60.0 \mathrm{ml} / \mathrm{min}$; vi) Eastern Cooperative Oncology Group PS of 02 ; and vii) patients provided written informed consent to participate in this study.

Exclusion criteria. The exclusion criteria were as follows: i) Severe uncontrollable complications, apart from malignant tumors (e.g., intestinal paralysis, pulmonary fibrosis, diabetes mellitus, heart failure, myocardial infarction, angina pectoris, renal failure, hepatic failure, mental disorders, cerebrovascular disorder and gastric/duodenal active ulcers); ii) symptomatic brain metastasis or clinical suspicion of brain metastasis; iii) convulsive disorder requiring treatment with anticonvulsants; iv) symptomatic ascites or pleural effusions requiring therapeutic paracentesis; v) pyloric stenosis or intestinal obstruction; vi) vomiting-related episodes or CTCAE v4.0 grade $\geq 2$ nausea; vii) history of hypersensitivity to palonosetron or ingredients of any other 5-HT3RA preparation; viii) history of hypersensitivity to ingredients of dexamethasone preparations; ix) pregnancy, lactation, and/ or refusal to practice contraception during the study period; $\mathrm{x}$ ) past history of palonosetron use; xi) incapable or reluctant to cooperate with the procedures necessary for this study; and xii) any patients who were deemed inappropriate to be subjects of this study, for any reason, by the investigator (or coinvestigator).

Medication administration. On day $1,0.75 \mathrm{mg} /$ body of palonosetron and $19.8 \mathrm{mg} /$ body of dexamethasone were dissolved in $100 \mathrm{ml}$ of physiological saline and administered intravenously over 15 min immediately prior to TC therapy. Dexamethasone in daily doses of $6.6 \mathrm{mg} /$ body in $100 \mathrm{ml}$ physiological saline was also administered intravenously on days 2 and 3 (Fig. 1).

Concomitant and contraindicated drugs. Dexamethasone preparations were contraindicated, unless otherwise specified, from $24 \mathrm{~h}$ prior to the administration of palonosetron until completion of the observation period. From $24 \mathrm{~h}$ prior to the administration of palonosetron until day 5, concomitant use of any of the following drugs with an antiemetic effect was prohibited, except for use as post-TC antiemetic treatment (rescue therapy): i) $\mathrm{NK}_{1}$ receptor antagonist antiemetics; ii) 5-HT3RA antiemetics; iii) all adrenocortical steroids except dexamethasone; iv) antidopaminergic drugs, such as metoclopramide and domperidone; v) phenothiazine antipsychotics, such as prochlorperazine and perphenazine; vi) antihistamines; vii) all benzodiazepines, except for the use of triazolam for insomnia as needed; and viii) other drugs, including haloperidol, droperidol, scopolamine and olanzapine. Concomitant use of serotonin-specific reuptake inhibitors and serotonin-norepinephrine reuptake inhibitors was also prohibited from $24 \mathrm{~h}$ prior to the administration of palonosetron through day 5 .

Statistical analysis. Logistic regression analysis was used for comparison of background variables. Inter-group or inter-subgroup differences were considered to be statistically significant when $\mathrm{P}<0.05$.

\section{Results}

Background variables. The data for background variables are presented in Table I. The median age was 60.5 years (range, 32-83 years) and the median body surface area was $1.44 \mathrm{~m}^{2}$ (range, $1.19-1.87 \mathrm{~m}^{2}$ ). The PS score was 0 in 34,1 in 4 and 2 in 4 patients. The diagnosis was uterine cervical cancer in 2 patients, endometrial cancer in 18 , ovarian cancer in 21 and triple cancer in 1 patient. Of the 42 patients, 29 received TC therapy as adjuvant chemotherapy and 13 as systemic chemotherapy. A total of 10 patients developed complications (diabetes mellitus in 4; hypertension in 3; thrombosis in 2; and arrhythmia, hyperlipidemia, insomnia and osteoarthritis of the hip in 1 patient each), whereas 32 patients had no concurrent disorders.

Response to palonosetron + dexamethasone therapy. For the acute, delayed and overall periods, the CR rates were 95.2, 
Table I. Patient characteristics.

Patient no. (\%)

Characteristics

$(n=42)$

Age (years)

Median (range)

Body surface area $\left(\mathrm{m}^{2}\right)$

Median (range)

$1.44(1.19-1.87)$

ECOG PS

0

$34(81.0)$

$4(9.5)$

1

4 (9.5)

Type of cancer

Cervical

Endometrial

Ovarian

$21(50.0)$

Others

Chemotherapy

Adjuvant

Systemic

Complications

Present

Absent

$32(76.2)$

ECOG PS, Eastern Cooperative Oncology Group performance status.

Table II. Complete response (CR) and complete control (CC) rate $(n=42)$.

\begin{tabular}{lcr}
\hline Period & CR rate, no. $(\%)$ & CC rate, no. $(\%)$ \\
\hline Acute & $40(95.2)$ & $38(90.5)$ \\
Delayed & $38(90.5)$ & $36(85.7)$ \\
Overall & $36(85.7)$ & $33(78.6)$
\end{tabular}

Table III. Rescue therapy for complete response (CR) and complete control (CC) cases.

\begin{tabular}{lcccc}
\hline Cases & $\begin{array}{c}\text { Acute period } \\
\text { (VAS) }\end{array}$ & $\begin{array}{c}\text { Rescue } \\
\text { therapy }\end{array}$ & $\begin{array}{c}\text { Delayed period } \\
\text { (VAS) }\end{array}$ & $\begin{array}{c}\text { Rescue } \\
\text { therapy }\end{array}$ \\
\hline 1 & 5 & Granisetron, dexamethasone & 1 & None \\
2 & 2 & Granisetron, dexamethasone & 0 & N/A \\
3 & 0 & N/A & 10 & Granisetron, dexamethasone \\
4 & 0 & N/A & 8 & Granisetron, dexamethasone \\
5 & 7 & None & 7 & Granisetron, dexamethasone, ramosetron \\
6 & 0 & N/A & 4 & Granisetron, dexamethasone \\
7 & 3 & None & 0 & N/A \\
8 & 0 & N/A & 3 & None \\
9 & 0 & N/A & 6 & None \\
\hline
\end{tabular}

VAS, visual analog scale; N/A, not applicable. 
Table IV. Subgroup analysis.

\begin{tabular}{|c|c|c|c|c|}
\hline Variables & $\mathrm{CR}$ rate $(\%)$ & Odds ratio & $95 \% \mathrm{CI}$ & P-value \\
\hline \multicolumn{5}{|l|}{ Age, years } \\
\hline$\geq 55(n=29)$ & 86.1 & 4.76 & $0.24-95.3$ & 0.005 \\
\hline$<55(n=13)$ & 100.0 & & & \\
\hline \multicolumn{5}{|l|}{$\mathrm{BSA}, \mathrm{m}^{2}$} \\
\hline$\geq 1.42(\mathrm{n}=26)$ & 92.3 & 0.59 & $0.09-3.84$ & 0.91 \\
\hline$<1.42(\mathrm{n}=16)$ & 87.5 & & & \\
\hline \multicolumn{5}{|l|}{ ECOG PS } \\
\hline $0(n=34)$ & 94.1 & 0.02 & $0.03-1.40$ & 0.36 \\
\hline $1-2(n=8)$ & 75.0 & & & \\
\hline \multicolumn{5}{|l|}{ Complications } \\
\hline Present $(n=10)$ & 80.0 & 3.59 & $0.53-24.3$ & 0.56 \\
\hline Absent $(n=32)$ & 93.8 & & & \\
\hline
\end{tabular}

BSA, body surface area; CR, complete response; CI, confidence interval.

Table V. Adverse events $(n=42)$.

\begin{tabular}{lcc}
\hline Toxicities & Grade1, no. $(\%)$ & Grade $\geq 2$, no. $(\%)$ \\
\hline Non-hematological & & 0 \\
Headache & $3(7.1)$ & 0 \\
Vertigo & $1(2.4)$ & $1(2.4)$ \\
Constipation & $14(33.3)$ & 0 \\
Abdominal pain & 0 & 0 \\
Hiccup & 0 & 0 \\
Allergy & 0 & 0 \\
Vascular pain & 0 & $1(2.4 \%)$ \\
Diarrhea & 0 & 0 \\
Hematological & 0 & 0
\end{tabular}

AST, aspartate aminotransferase; ALT, alanine aminotransferase, $\gamma$-GTP; $\gamma$-glutamyltransferase; ALP, alkaline phospatase.

90.5 and $85.7 \%$, respectively, whereas the CC rates were 90.5 , 85.7 and $78.6 \%$, respectively (Table II). The severity grades of nausea during the acute, delayed and overall periods were $0.45 \pm 1.38,1.13 \pm 2.40$, and $0.79 \pm 1.99$, respectively. Episodes of nausea occurred in 4 patients during the acute phase, 7 during the delayed period and 9 during the overall period. The mean severity grades of nausea for the patients with this adverse event during the periods investigated in this study were $3.17 \pm 2.19,3.96 \pm 2.99$, and $3.69 \pm 2.77$, respectively (data not shown).

Rescue therapy. Rescue therapy was administered to 2, 4, and 6 patients during the acute, delayed and overall periods, respectively. Granisetron and dexamethasone were prescribed for this purpose in the 2 patients with nausea during the acute period. Granisetron and dexamethasone were used for this purpose during the delayed period in all 4 affected patients, whereas 1 patient who responded poorly also received an additional ramosetron regimen (Table III).
Subgroup analysis. The subgroup analysis results are summarized in Table IV. Higher delayed period CR rates were recorded for the following subgroups: Age $<55$ years, body surface area $\geq 1.47 \mathrm{~m}^{2}$, PS score 0 and absence of complications.

Adverse events. Adverse event data are presented in Table V. As regards non-hematotoxic adverse events, grade $\geq 2$ constipation and diarrhea occurred in 1 patient $(2.4 \%)$ each. No grade $\geq 3$ hematotoxicities were identified when the blood biochemical test parameters were measured.

\section{Discussion}

It is generally recognized that, among the adverse reactions to treatment with antineoplastic drugs, nausea and vomiting are the most disagreeable symptoms suffered by patients (9). Persistent nausea and vomiting may lead to dehydration, electrolyte disturbances and malnutrition, whereas patient 


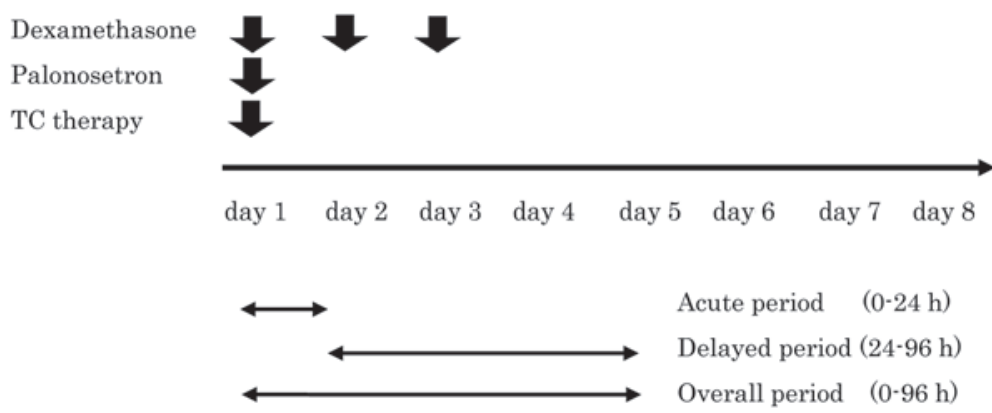

Figure 1. Treatment protocol. On day $1,0.75 \mathrm{mg} /$ body of palonosetron and $19.8 \mathrm{mg} /$ body of dexamethasone were dissolved in $100 \mathrm{ml}$ of physiological saline and administered intravenously over $15 \mathrm{~min}$ immediately prior to TC therapy. Dexamethasone in daily doses of $6.6 \mathrm{mg} / \mathrm{body}$ in $100 \mathrm{ml}$ physiological saline was also administered intravenously on days 2 and 3. TC, paclitaxel and carboplatin.

willingness to undergo cancer treatment diminishes in the presence of severe CINV. Therefore, control of nausea and vomiting is considered to be crucial for the continuation of cancer treatment by maintaining the patient's general condition and QOL. Previously, antiemetic therapy for CINV consisted solely of corticosteroids, with a rate of successful acute CINV control of $\sim 30 \%$ (10). The CINV control rate increased to $\sim 70 \%$ with the advent of first-generation 5-HT3RA medications (11). The first-generation 5-HT3RAs proved to be effective in the control of acute nausea and vomiting; however, a proportion of patients suffer delayed nausea and vomiting, which constitutes a major problem in cancer chemotherapy. In 2010, palonosetron, a second-generation 5-HT3RA, was approved in Japan. The efficacy of palonosetron in controlling delayed as well as acute nausea and vomiting is attributed to its long plasma elimination half-life (40 h) and strong affinity for 5-HT3 receptors.

There have been several reports demonstrating an antiemetic effect of palonosetron on patients receiving highly emetogenic chemotherapeutic agents (HEC) $(12,13)$. As regards moderate emetogenic chemotherapeutic agents (MEC), however, the majority of the studies have focused on antiemetic therapy regimens including an anthracycline classified under HEC in the National Comprehensive Cancer Network guidelines combined with cyclophosphamide $(14,15)$. However, the number of studies assessing the efficacy of palonosetron in a combined antiemetic regimen in patients treated with MEC alone is currently limited. Furthermore, no studies have yet assessed the antiemetic effect of palonosetron on delayed-onset nausea and vomiting in gynecological cancer patients treated with MEC. Thus, we conducted this phase II clinical trial to assess the efficacy and safety of palonosetron + dexamethasone in patients receiving TC therapy.

In the PROTECT study, comparing a combined regimen of the first-generation 5-HT3RA granisetron and dexamethasone with a combined regimen of the second-generation 5-HT3RA palonosetron and dexamethasone, there was no improvement with respect to acute nausea induced by HECs, such as cisplatin or doxorubicin/cyclophosphamide, frequently used in breast cancer cases; however, the results confirmed a significantly higher efficacy of palonosetron compared to granisetron in controlling delayed nausea and vomiting (13).

We evaluated nausea and vomiting using the MASCC Antiemesis Tool in the present clinical trial, utilizing VAS for nausea and in terms of the frequency of vomiting episodes. The majority of the previous clinical studies evaluated the severity of nausea indirectly, according to whether or not any rescue therapy was undertaken, rather than directly. The use of VAS allowed the objective assessment of the severity of nausea in this study, as the severity of nausea depends on the patient's (subjective) viewpoint.

TC therapy is classified into the MEC category. The present data demonstrated high $\mathrm{CR}$ and $\mathrm{CC}$ rates for delayed vomiting in patients receiving TC therapy with concomitant palonosetron + dexamethasone regimen.

In patients who failed to attain $\mathrm{CR}$ and $\mathrm{CC}$, reduction of nausea was achieved with granisetron, dexamethasone or ramosetron, which are agents with different mechanisms of action. A phase III clinical trial designed to compare palonosetron + dexamethosone vs. granisetron, or palonosetron + dexamethasone vs. aprepitant, is required to determine whether palonosetron + dexamethasone exerts a prophylactic effect against nausea in patients receiving TC therapy. Such a clinical trial is currently being planned by the Japanese Gynecologic Oncology Group.

Young patients are reportedly at high risk of CINV $(16,17)$. The present subgroup analysis revealed a significantly higher $\mathrm{CR}$ rate in women aged $<55$ years compared to that in women aged $\geq 55$ years, in terms of treatment of delayed-onset CINV. Previous studies have focused on the use of first-generation 5-HT3RA, while the present data suggest the potential efficacy of the second-generation 5-HT3RA palonosetron in combination with dexamethasone for the control of delayed-onset vomiting, which reportedly occurs more frequently among younger patients. Furthermore, the present analysis revealed marginally higher CR rates for the following subgroups: Body surface area $\geq 1.47 \mathrm{~m}^{2}$, PS score 0 , adjuvant chemotherapy and absence of complications, although the differences did not reach statistical significance.

Constipation occurred as an adverse event in 16 patients (38.1\%), but was rated as $\geq$ grade 2 in only 1 patient $(2.4 \%)$. It is a general rule at our facility that all the patients receive oral laxative medications following gynecological cancer surgery; thus, none of the patients in the present series experienced serious constipation. Headache occurred in 3 patients (7.1\%), vertigo in $1(2.4 \%)$ and diarrhea in 2 patients $(4.8 \%)$; the causes for these adverse events remain unclear. No grade $\geq 3$ hematotoxicity was observed in the blood biochemical tests. Thus, the adverse reactions experienced were considered to be within acceptable limits and were thus considered to have been effectively controlled. 
The present phase II clinical trial demonstrated the efficacy of palonosetron + dexamethasone in preventing delayed-onset nausea in gynecological cancer patients receiving TC therapy. This combined antiemetic regimen was associated with only mild adverse reactions and may serve as supportive therapy, allowing cancer chemotherapy to be continued while maintaining the patients' QOL.

\section{References}

1. Sekine I, Segawa Y, Kubota K and Saeki T: Risk factors of chemotherapy-induced nausea and vomiting: index for personalized antiemetic prophylaxis. Cancer Sci 104: 711-717, 2013

2. Richardson JL, Marks G and Levine A: The influence of symptoms of disease and side effects of treatment on compliance with cancer therapy. J Clin Oncol 6: 1746-1752, 1988.

3. Kaizer L, Warr D, Hoskins P, Latreille J, Lofters W, Yau J, Palmer M, Zee B, Levy M, Pater J. Effect of schedule and maintenance on the antiemetic efficacy of ondansetron combined with dexamethasone in acute and delayed nausea and emesis in patients receiving moderately emetogenic chemotherapy: a phase III trial by the National Cancer Institute of Canada Clinical Trials Group. J Clin Oncol 12: 1050-1057, 1994.

4. Yang LP and Scott LJ: Palonosetron: in the prevention of nausea and vomiting. Drugs 69: 2257-2278, 2009.

5. Curran MP and Robinson DM: Aprepitant: a review of its use in the prevention of nausea and vomiting. Drugs 69: 1853-1878, 2009.

6. MASCC antiemesis tool (MAT): http://www.mascc.org/mat. Accessed March 3, 2010.

7. NCCN Clinical Practice Guidelines in OncologyAntiemesis- ver.4 2009. http://www.nccn.org/professionals/ physician_gls/f_guidelines.asp\#supportive. Accessed March 3, 2010.

8. National Cancer Institute (2009). Common Terminology Criteria for Adverse Events v4.0. NCI, NIH, DHHS. Available from: https://wiki.nci.nih.gov/display/VKC/Common + Terminology + Criteria + for + Adverse +Events + FAQ \# Common Terminology Criteria for Adverse Events FAQ - What is the official reference citation for CTCAE v40. Accessed May 29, 2009.

9. Aapro MS: Palonosetron as an anti-emetic and anti-nausea agent in oncology. Ther Clin Risk Manag 3: 1009-1020, 2007.
10. Ioannidis JP, Hesketh PJ and Lau J: Contribution of dexamethasone to control of chemotherapy-induced nausea and vomiting: a meta-analysis of randomized evidence. J Clin Oncol 18: 3409-3422, 2000

11. Olver I, Paska W, Depierre A, Seitz JF, Stewart DJ, Goedhals L, McQuade B, McRae J and Wilkinson JR: A multicentre, double-blind study comparing placebo, ondansetron and ondansetron plus dexamethasone for the control of cisplatin-induced delayed emesis. Ondansetron Delayed Emesis Study Group. Ann Oncol 7: 945-952, 1996

12. Aapro MS, Grunberg SM, Manikhas GM, Olivares G, Suarez T, Tjulandin SA, Bertoli LF, Yunus F, Morrica B, Lordick F and Macciocchi A: A phase III, double-blind, randomized trial of palonosetron compared with ondansetron in preventing chemotherapy-induced nausea and vomiting following highly emetogenic chemotherapy. Ann Oncol 17: 1441-1449, 2006.

13. Saito M, Aogi K, Sekine I, Yoshizawa H, Yanagita Y, Sakai H, Inoue K, Kitagawa C, Ogura T and Mitsuhashi S: Palonosetron plus dexamethasone versus granisetron plus dexamethasone for prevention of nausea and vomiting during chemotherapy: a double-blind, double-dummy, randomized, comparative phase III trial. Lancet Oncol 10: 115-124, 2009.

14. Eisenberg P, Figueroa-Vadillo J,Zamora R, Charu V, Hajdenberg J, Cartmell A, Macciocchi A and Grunberg S; 99-04 Palonosetron Study Group: Improved prevention of moderately emetogenic chemotherapy-induced nausea and vomiting with palonosetron, a pharmacologically novel 5-HT3 receptor antagonist: results of a phase III, single-dose trial versus dolasetron. Cancer 98: 2473-2482, 2003

15. Gralla R, Lichinitser M, Van der Vegt S, Sleeboom H, Mezger J, Peschel C, Tonini G, Labianca R, Macciocchi A and Aapro M: Palonosetron improves prevention of chemotherapy-induced nausea and vomiting following moderately emetogenic chemotherapy: results of a double-blind randomized phase III trial comparing single doses of palonosetron with ondansetron. Ann Oncol 14: 1570-1577, 2003.

16. Hesketh PJ: Chemotherapy-induced nausea and vomiting. N Engl J Med 358: 2482-2494, 2008

17. Hesketh PJ, Aapro M, Street JC and Carides AD: Evaluation of risk factors predictive of nausea and vomiting with current standard-of-care antiemetic treatment: analysis of two phase III trials of aprepitant in patients receiving cisplatin-based chemotherapy. Support Care Cancer 18:1171-1177, 2010. 